\title{
Factores psicosociales en los pacientes con úlceras venosas y su asociación con la cicatrización
}

\author{
Psychosocial factors of patients with venous leg ulcers and their \\ association with healing
Fatores psicossociais de pacientes com úlceras venosas e sua associação com a cicatrização

Johana Enyd Cifuentes Rodriguez1,", Sandra Guerrero Gamboa ${ }^{2}$

ORCID IDS

Cifuentes JE (D) https://orcid.org/0000-0001-5384-3480

Guerrero SG (D) https://orcid.org/0000-0002-0893-0478

\section{CÓMO CITAR}

Cifuentes, JE, Guerrero, SG. Factores psicosociales en los pacientes con úlceras venosas y su asociación con la cicatrización. ESTIMA, Braz. J. Enterostomal Ther., 18: e0720, 2020. https://doi.org/10.30886/ estima.v18.845_ESP

\begin{abstract}
RESUMEN
Objetivo: Identificar los factores psicosociales presentes en pacientes con úlceras venosas y la evidencia disponible sobre la asociación que estos factores tienen con la curación de este tipo de heridas. Métodos: Revisión integradora de la literatura de estudios cuantitativos en las bases de datos MEDLINE, Scielo y Cochrane Library entre los años 2008 y 2019 , utilizando las palabras clave, factores psicosociales, úlcera venosa, cicatrización de heridas ansiedad y depresión en idioma inglés, español y portugués. Resultados: Dieciséis estudios fueron incluidos. Los factores psicosociales presentes en los pacientes con úlceras venosas fueron depresión, ansiedad, sentimientos de impotencia, bienestar subjetivo, autoestima, soledad y espiritualidad. El estrés, una percepción negativa de la úlcera venosa, vivir solo y la experiencia severa de síntomas como dolory depresión tienen asociaciones estadísticamente significativas con periodos más prolongados de curación. Conclusión: La depresión es uno de los factores psicológicos medido y presente con mayor frecuencia en esta población. La evidencia disponible frente a la asociación de los factores psicosociales con la curación de úlceras venosas es escasa.
\end{abstract}

DESCRIPTORES: Úlcera varicosa. Psicología. Emociones. Cicatrización de heridas. Enfermería. Estomaterapia.

\begin{abstract}
Objectives: To identify psychosocial factors present in patients with venous leg ulcers and the association that these factors have in the healing of venous leg ulcers. Methods: An integrative review of the quantitative studies in MEDLINE, Scielo and Cochrane Library databases, between 2008 and 2019, using the keywords, psychosocial factors, venous ulcer, wound healing, anxiety and depression in English, Spanish and Portuguese. Results: sixteen studies were included. The psychosocial factors present in patients with venous ulcers were depression, anxiety, feelings of helplessness, subjective well-being, self-esteem, loneliness and spirituality. Stress, a negative perception of venous ulcer, living alone and severe experience of symptoms such as pain and depression have statistically significant associations with longer periods of healing. Conclusions: Depression is one of the most frequently measured factors and present in this population. The available evidence on the association of psychosocial factors with the healing of venous ulcers is low.
\end{abstract}

DESCRIPTORS: Varicose ulcer. Psychology. Emotions. Wound healing. Nursing. Enterostomal therapy.

1. Universidad Nacional de Colombia, Facultad de Enfermería, Programa Doctorado en Enfermería - Bogotá, Colombia

*Autor correspondiente: jecifuentesr@unal.edu.co

Recibido: Feb. 7, 2020 | Aceptado: Mar. 27, 2020 


\section{RESUMO}

Objetivo: Identificar os fatores psicossociais presentes em pacientes com úlceras venosas e as evidências disponíveis sobre a associação que esses fatores têm com a cicatrização desse tipo de ferida. Métodos: Revisão integrativa da literatura de estudos quantitativos nas bases de dados, MEDLINE, Scielo e Cochrane Library entre 2008 e 2019, utilizando as palavras-chave, fatores psicossociais, úlcera venosa, ansiedade e depressão em feridas em inglês, espanhol e português. Resultados: Dezesseis estudos foram incluídos. Os fatores psicossociais presentes nos pacientes com úlceras venosas foram depressão, ansiedade, sentimentos de desamparo, bemestar subjetivo, autoestima, solidão e espiritualidade. O estresse, a percepção negativa da úlcera venosa, o fato de morar sozinho e a experiência severa de sintomas como dor e depressão têm associações estatisticamente significativas com períodos mais longos de cicatrização. Conclusões: A depressão é um dos fatores psicológicos medidos e mais frequentemente presentes nessa população. As evidências disponíveis sobre a associação de fatores psicossociais com a cicatrização de úlceras venosas são baixas.

DESCRITORES: Úlcera Varicosa. Psicologia. Emoções. Cicatrização. Enfermagem, Estomaterapia.

\section{INTRODUCCIÓN}

Las úlceras venosas (UV) constituyen entre el 75\% y $80 \%$ de la totalidad de úlceras en miembros inferiores. Son la manifestación clínica más avanzada de la hipertensión venosa ambulatoria de larga evolución, debido al reflujo venoso por incompetencia valvular y obstrucción venosa ${ }^{1}$. Se estima que las UV afectan entre el 1\% al 3\% de la población mundial, la prevalencia en España es del 0.5\% al 0.8\% la cual se incrementa con la edad, siendo del 3-5\% en mayores de 65 años y su incidencia es de 2 y 5 nuevos casos por mil personas año ${ }^{2,3}$. Respecto al contexto Latinoamericano no existen suficientes estudios epidemiológicos. Sin embargo, un consenso de esta región suramericana estimo una prevalencia de ulceras venosas entre $3 \%$ y $6 \%{ }^{4}$.

Aproximadamente el 50\% de las UV son crónicas, es decir, son heridas que no siguen un proceso de reparación ordenado para la curación oportuna de la lesión anatómica o funcional, están presentes durante más de seis semanas, incluso años y tienen un carácter cíclico, con periodos de curación seguidos de recurrencia ${ }^{5}$. En promedio, las UV están presentes durante 9 meses, el 93\% sanará en 12 meses y el 7\% restante permanecerá sin curar después de 5 años, con una tasa de recurrencia de hasta el 70\% dentro de los tres meses posteriores a la curación ${ }^{6}$. Aspectos que la convierte en una condición crónica compleja, que requiere de automanejo. El automanejo desde el modelo conceptual de Enfermería del Automanejo Individual y Familiar de la Universidad de Yale, se refiere a la capacidad del individuo, en conjunto con la familia, la comunidad y los profesionales de la salud, para manejar los síntomas, los tratamientos, los cambios en el estilo de vida y las consecuencias psicosociales, culturales y espirituales de las condiciones de salud ${ }^{7}$. De manera que, las
UV como cualquier otra condición crónica demanda cuidados de enfermería prolongados, significativos cambios de estilo de vida, adherencia a regímenes terapéuticos que incluyen, uso de terapia compresiva, estrategias de mantenimiento y preventivas que favorecen la curación de UV y previenen su recurrencia como, elevación de las piernas, control de peso, actividad física, cuidado de la piel, protección contra lesiones, manejo de las emociones, recibir soporte social, entre otras ${ }^{3,8}$.

Bajo esta perspectiva conceptual, es necesario identificar primero, aquellos factores que pueden comportarse como facilitadores y barreras en el automanejo de cierta condición, en este caso las UV, ver Fig. 1. Dentro de este grupo de factores, se encuentran los factores psicosociales (características psicológicas y sociales) entendidos como aquellos sentimientos, emociones, pensamientos, actitudes y aspectos sociales, como el soporte social, que pueden influir tanto en los resultados proximales y distales del automanejo, los cuales involucran el comportamiento, la cognición, biomarcadores, manejo de los síntomas, calidad de vida, acceso a los servicios de salud, un mejor estado psicosocial, entre otros.

Dentro de los postulados de este marco conceptual se señala que, es posible modificar estos resultados si se modifican los factores que actúan como facilitadores y barreras, esto dependerá de las necesidades de los pacientes, o también se puede fortalecer el proceso de automanejo, activando recursos psicológicos como la autoeficacia, el afrontamiento, la comunidad, procesamiento de emociones, por nombrar algunos ${ }^{7}$. Por ejemplo, para el caso de las UV, Finlayson ${ }^{9}$ utilizó este marco conceptual para identificar aquellos factores de riesgo y protectores que influyen en la recurrencia de UV y halló que, la edad, los antecedentes de: trombosis venosa profunda, de múltiples UV previas y la duración total de la UV fueron factores de riesgo para 


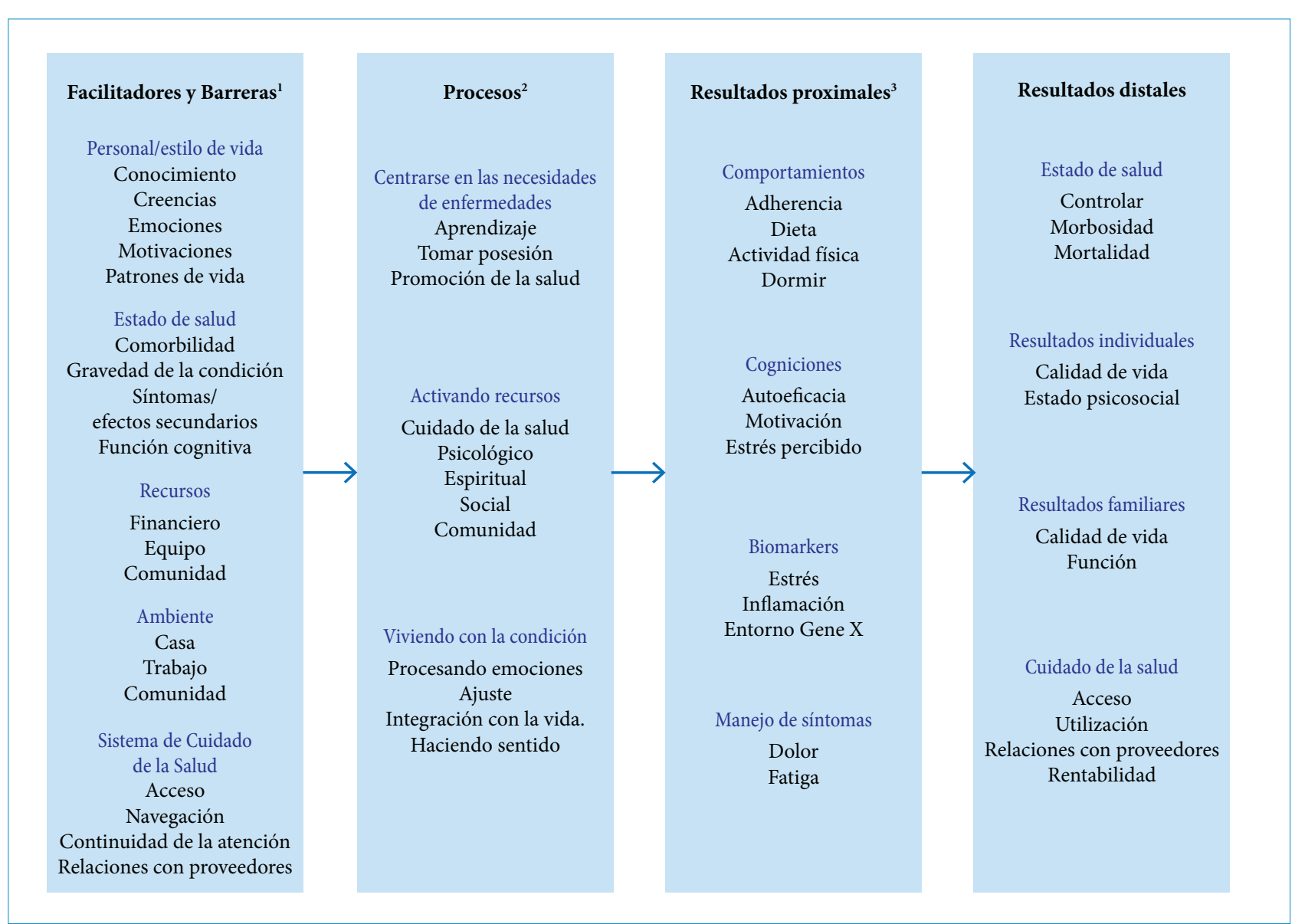

Figura 1. Marco conceptual del Automanejo Individual y Familiar.

la recurrencia de las úlceras. Mientras que, la elevación de las piernas, la marcha y la autoeficacia fueron los factores protectores que mejoraron la probabilidad de prevenir la recurrencia de estas. Otros estudios han demostrado que los pacientes con ansiedad, depresión y enfermedad coronaria como antecedente, aumentan los tiempos de cicatrización y tienen mayor riesgo de recurrencia del UV ${ }^{10,11}$. Especificar cuáles factores psicosociales están presentes en los pacientes con UV es difícil ya que desde hace poco tiempo se incluyen tanto en el estudio de los factores de riesgo para desarrollar UV como en el estudio de los factores que influyen en su recurrencia, ya que ha primado el componente fisiopatológico sobre los aspectos psicológicos y sociales de las personas. No obstante, la ansiedad y la depresión son los más comunes.

Por otra parte, revisiones sistemáticas de la literatura ${ }^{12,13}$ sobre el impacto que las UV tienen en la calidad de vida de los pacientes y síntesis de evidencia cualitativa sobre la experiencia de vivir con esta condición han permitido dilucidar las consecuencias psicológicas y sociales que las UV y sus síntomas asociados como, dolor, exudado, edema, fatiga y trastornos del sueño, generan. De hecho, recientemente los denominados síntomas psiconeurológicos que incluyen, dolor, disfunción cognitiva, fatiga, depresión y ansiedad asociados con las UV crónicas esta tomando fuerza debido a que estos síntomas pueden afectar el procesamiento mental y emocional de las personas, necesarios para el automanejo de su enfermedad ${ }^{14}$. Esto es, estos síntomas pueden generar nuevos estados emocionales negativos o exacerbar los actuales.

Entre las consecuencias emocionales de las UV se hallan la ansiedad, depresión, vergüenza, baja autoestima, pérdida de control de sus propios cuerpos, impotencia y desesperanza; entre las sociales, vivir una vida restringida, perdida de trabajo, aislamiento social, desconexión social, soledad y menos soporte social percibido ${ }^{12,13,15}$. Aspectos que a su vez también pueden tener un impacto en los procesos de cicatrización de los pacientes con UV, pues múltiples estudios han demostrado que, el estrés y las emociones negativas como ansiedad y depresión, interrumpen los procesos de cicatrización de las heridas al mediar los ejes medulares hipotalámicos-hipofisiarios-suprarrenales y simpáticos-suprarrenales, prolongando la etapa inflamatoria e inhibiendo la proliferación de fibroblastos y queratinocitos, 
evitando así que las heridas se curen ${ }^{16}$. Por ejemplo, Bosch et $\mathrm{al}^{17}$ y Doering et $\mathrm{al}^{18}$, investigaron la asociación entre síntomas depresivos y cicatrización de heridas. El primero, a través de heridas estandarizadas realizadas en el paladar duro de 193 adultos jóvenes identificó que, puntajes más altos de depresión y un nivel alto de disforia se asoció con una cicatrización tardía, heridas más grandes y 3.5 más probabilidades de presentar una tasa de curación más lenta que la mediana. El segundo, en una muestra de pacientes sometidos a revascularización cardiaca halló una asociación entre síntomas depresivos, deterioro en la cicatrización de sus heridas operatorias, infección y una baja recuperación física y emocional. Cole-King y Harding ${ }^{19}$ señalaron que, la curación tardía de pacientes con úlceras crónicas en miembros inferiores también se relacionó con niveles altos de ansiedad y depresión. Otros factores asociados significativamente con un retraso en la curación de UV fueron la baja clase social, falta de calefacción central en el hogar, ser soltero y tener un bajo soporte social ${ }^{20}$.

Teniendo en cuenta que, (a) en la actualidad se reconoce la influencia de algunos factores psicosociales en la curación de las heridas, (b) la investigación cuantitativa de evaluación/ detección de estos factores en la población con UV se ha incrementado en los últimos años, así como el estudio de su posible impacto en la curación y (c) considerando que solo algunos documentos de consenso y guías de práctica clínica internacionales para la atención de enfermería a pacientes con UV mencionan someramente la valoración de dichos factores $^{1,21,22}$, lo cual tiene implicaciones en el cuidado de enfermería planeado y proporcionado a estos pacientes.

En consecuencia, el objetivo de esta revisión es identificar basados en estudios cuantitativos, cuáles son los factores psicosociales presentes en los pacientes con UV y la asociación que estos factores tienen en la curación de este tipo de heridas.

\section{MÉTODOS}

Se realizó una revisión integradora de la literatura, este método de investigación permite la búsqueda, evaluación crítica y síntesis de la evidencia disponible sobre un fenómeno de interés con el fin de vislumbrar el estado actual del conocimiento al respecto. De esta forma, se desarrolló siguiendo los pasos propuestos por Baldini Soares y colaboradores: identificación del tema y selección de la pregunta de investigación; establecer los términos de búsqueda de los estudios, establecimiento de criterios de inclusión y exclusión; selección los estudios de acuerdo a los criterios establecidos, clasificación de la calidad de los estudios en relación con el nivel de evidencia, extracción y síntesis de la información y discusión de los hallazgos ${ }^{23}$.

En consecuencia, dos fueron las preguntas que orientaron la presente revisión: ¿Cuáles son los factores psicosociales presentes en los pacientes con UV reportados en la literatura? y ¿Cuál es la evidencia disponible sobre la asociación que estos factores tienen en la curación de las UV? Acto seguido, se elaboró una estrategia de búsqueda mediante combinaciones de los siguientes descriptores en los idioma inglés, español y portugués: venous leg ulcer, psychosocial factor, psychology, wound healing, emotions, utilizando los operadores booleanos "AND” “'OR”; en las bases de datos, Scielo, MEDLINE y Cochrane Library. Los criterios de inclusión considerados fueron: a) artículos publicados entre los años 2008 y 2019 en idioma inglés, portugués y español, b) estudios cuya muestra incluyera personas con úlceras de etiología venosa y c) estudios cuantitativos cuyo objetivo fuera identificar o conocer algún factor psicosocial presente en esta población y la asociación entre estos y la curación de estas heridas específicamente. Los criterios de exclusión fueron: a) revisiones de la literatura, artículos de opinion, editorias y literatura gris y c) estudios desarrollados en animales. La búsqueda fue conducida electrónicamente durante abril-mayo de 2019 y actualizada en diciembre de 2019.

Posteriormente, dos revisores de forma independiente realizaron lectura crítica de los estudios seleccionados de acuerdo con los criterios propuestos por Lobiondo \& Haber ${ }^{24}$ los cuales evalúan coherencia interna (antecedentes del problema, pregunta de investigación y objetivos) y coherencia metodológica, fiabilidad, validez interna-externa, credibilidad y utilidad de los resultados para la práctica de enfermería.

\section{Extracción y síntesis de datos}

Los datos de los estudios se extrajeron y organizaron en una tabla (Tab. 1) que incluyó autores, año, país, objetivo (s), diseño, muestra, instrumentos utilizados para medir los factores psicológicos incluidos, hallazgos principales y nivel de evidencia, la clasificación de acuerdo a su nivel de evidencia se hizo de acuerdo a los criterios propuestos por el Instituto Joanna Briggs $(\mathrm{IJB})^{25}$. Esta etapa del proceso fue realizada por dos autores de forma independiente y revisada por un tercer investigador con experiencia cuando fue necesario. Seguidamente, se realizó una síntesis narrativa para analizar los datos extraídos y se presentan a continuación junto con la discusión. 


\section{RESULTADOS}

De acuerdo con los objetivos de esta revisión, los resultados se sintetizan en términos de resultados de búsqueda, descripción de los estudios, factores psicosociales en los pacientes con UV, asociación de factores psicosociales con la curación de las UV e implicaciones para la práctica de enfermería.

\section{Resultados de la búsqueda}

La búsqueda inicial arrojó 335 artículos, se eliminaron 132 por duplicación y 203 se retuvieron. Después de revisar y seleccionar la relevancia de los títulos y resúmenes, los autores descartaron 176 artículos según los criterios descritos, en su mayoría se excluyeron porque la población de estudio no correspondía a pacientes con UV, no trataban algún factor psicosocial, no eran estudios de investigación y dentro de las variables contempladas para determinar la curación de UV no se incluyó ningún aspecto psicosocial. En consecuencia, 16 artículos cumplieron con los criterios de inclusión y fueron incluidos en la presente revisión, ver Fig. 2.

\section{Descripción de los estudios}

Esta revisión incluyó 16 estudios, siete observacionales de tipo analítico y nueve descriptivos transversales. El nivel de evidencia de los estudios fue predominante IV de acuerdo a la clasificación propuesta por el $\mathrm{IJB}^{25}$. Estos estudios fueron conducidos en Brasil (50\%; $n=8)$, Inglaterra (12.5\%; $n=2)$, Australia (18.75\%; n=3), Estados Unidos (6.25\%;n=1), Francia $(6.25 \% ; n=1)$ y Grecia(6.25;n=1).

Se llevaron a cabo en su mayoría en servicios de consulta externa, clínicas de heridas comunitarias y servicios de hospitalización. Cuatro estudios buscaron identificar la posible asociación entre algunos factores psicosociales y la curación tardía de las úlceras venosas ${ }^{26-29}$, seis estudios midieron el nivel de depresión de estos paciente ${ }^{24-29}$ y los estudios restantes evaluaron otros factores como espiritualidad, esperanza, impotencia, bienestar subjetivo, autoestima e imagen corporal y su relación con algunas características sociodemográficas. Un total de 1738 pacientes participaron en los 16 estudios identificados. La mayoría de los participantes fueron mujeres y las edades oscilaron entre 20 a 70 años. En el periodo de 2013 a 2016 se concentró el 62.5\%(n=10/16) de la producción científica en este tema. La Tab. 1 resume las características de los artículos revisados y sus principales hallazgos.

Respecto a la lectura crítica de los estudios todos presentaron coherencia frente a la descripción de sus áreas problemas, pregunta de investigación y objetivos, sin embargo, algunos estudios no reportaron pruebas de validez de los instrumentos utilizados para medir los factores psicosociales analizados lo que podría limitar la validez de sus hallazgos. El hecho que la mayoría de los estudios utilizaron muestras a conveniencia limita la interpretación y la generalización de los resultados.

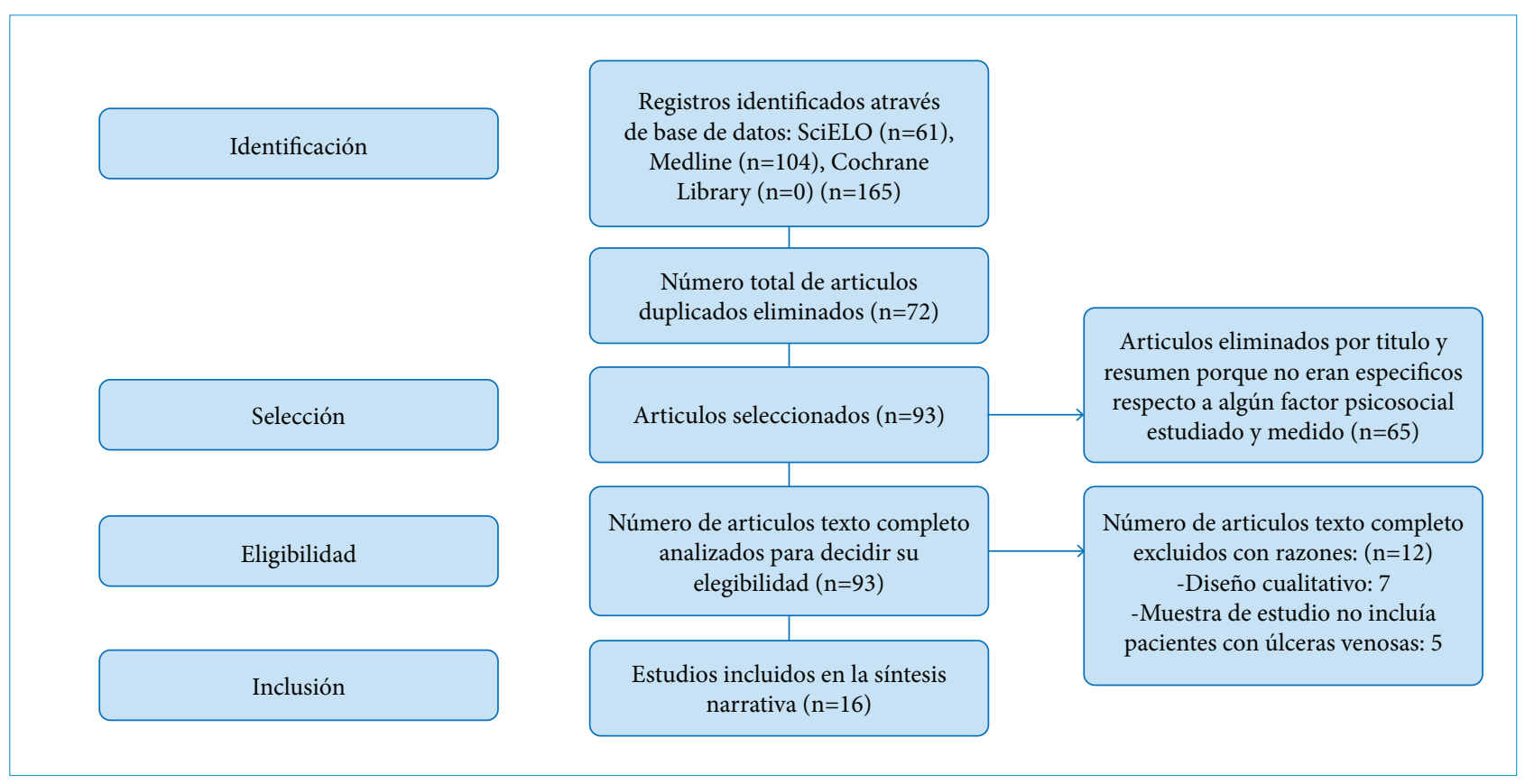

Figura 2. Flujograma del proceso de búsqueda y selección de artículos. 
Tabla 1. Factores psicosociales en pacientes con úlceras venosas, descripción de los estudios.

\begin{tabular}{cccc}
\hline Autores, & & & Diseño \\
año, país & Objetivo(s) & Hallazgos & principales \\
y nivel de & muestra & Instrumentos & \\
evidencia & & & \\
\hline
\end{tabular}

Los niveles de depresión fueron significativamente mayores en el grupo de

Identificar deficiencias en la salud psicológica Moffat et $a^{30}$. de pacientes con úlcera (2009) Inglaterra III venosa en comparación con una muestra de la población general de la misma edad y sexo.
- Nottingham Health Profil (caliad de vida) Estudio de casos - Hospital Anxiety and y controles Depression Scale - The Medical Outcomes 95 pacientes Study Social Support Survey The COPE scale casos (media de 5.3 vs. 3.6, $\mathrm{P}<0$. 001) El apoyo social fue significativamente menor en los casos que en los controles ( $P \leq .008)$ y los casos también usaron significativamente menos estrategias de afrontamiento que los controles. Los casos presentaron una calidad de vida significativamente más deficiente en todos los dominios en comparación con los controles $(P \leq 0.001)$.

\begin{tabular}{|c|c|c|c|}
\hline $\begin{array}{l}\text { Souza et } \mathrm{al}^{31} \text {. } \\
(2009) \\
\text { Brasil } \\
\text { IV }\end{array}$ & $\begin{array}{l}\text { Evaluar la presencia de } \\
\text { ansiedad y depresión en } \\
\text { pacientes con úlceras } \\
\text { venosas e investigar sus } \\
\text { posibles asociaciones } \\
\text { con variables } \\
\text { sociodemográficas. }\end{array}$ & $\begin{array}{l}\text { Estudio } \\
\text { transversal } \\
\text { prospectivo y } \\
\text { aleatorio. } \\
30 \text { pacientes }\end{array}$ & $\begin{array}{l}\text { Hospital Anxiety and } \\
\text { Depression Scale }\end{array}$ \\
\hline $\begin{array}{l}\text { Faria et } \text { al }^{32} \text {. } \\
(2011) \\
\text { Brasil } \\
\text { IV }\end{array}$ & $\begin{array}{l}\text { Evaluar la calidad de } \\
\text { vida, autoestima y } \\
\text { estado funcional de } \\
\text { pacientes con úlceras } \\
\text { venosas crónicas. }\end{array}$ & $\begin{array}{l}\text { Estudio } \\
\text { descriptivo } \\
\text { con grupo } \\
\text { comparativo. } \\
80 \text { pacientes } \\
\text { con úlceras } \\
\text { venosas y } 80 \\
\text { pacientes sin } \\
\text { úlceras venosas. }\end{array}$ & $\begin{array}{l}\text { - The Medical Outcomes } \\
\text { Study 36-Item Short } \\
\text { Form Health Survery } \\
\text { questionnaire (SF-36) } \\
\text { - The Rosenberg Self- } \\
\text { Esteem scale } \\
\text { - Standford } \\
\text { Health Assessment } \\
\text { Questionnaire (HAQ-20), } \\
\text { short version. }\end{array}$ \\
\hline
\end{tabular}

Se identificó ansiedad en el 30\% de los pacientes y depresión en el 40\%. No se encontró una asociación estadísticamente significativa entre la ansiedad o la depresión y las variables sociodemográficas.

Se hallaron diferencias significativas entre los grupos respecto a la calidad de vida en las dimensiones de funcionamiento físico, de rol, dolor corporal, funcionamiento social, rol emocional, vitalidad y estado general de salud $(P=<0.005)$. No hubo diferencias significativas respecto al autoestima dentro de los participantes. También hubo diferencias significativas frente al estado funcional en todas las categorías ( $P=0.001$ ) excepto en la categoría alimentación.

\begin{tabular}{|c|c|c|c|c|}
\hline $\begin{array}{l}\text { Salomé } \\
\text { et al } \\
\text { (2012) } \\
\text { Brasil } \\
\text { IV }\end{array}$ & $\begin{array}{l}\text { Evaluar el nivel de } \\
\text { síntomas depresivos } \\
\text { presentados por los } \\
\text { pacientes con úlcera } \\
\text { venosa. }\end{array}$ & $\begin{array}{l}\text { Estudio } \\
\text { descriptivo } \\
\text { transversal. } \\
60 \text { pacientes }\end{array}$ & $\begin{array}{l}\text { Beck Depression } \\
\text { Inventoy }\end{array}$ & $\begin{array}{l}\text { Los cinco síntomas más frecuentes fueron } \\
\text { Tristeza, distorsión de la imagen corporal, } \\
\text { disminución de la libido, aislamiento social } \\
\text { y auto depreciación. }\end{array}$ \\
\hline $\begin{array}{l}\text { Salomé } \\
\text { et al }{ }^{34} \text {. } \\
\text { (2013) } \\
\text { Brasil } \\
\text { IV }\end{array}$ & $\begin{array}{l}\text { Evaluar el bienestar } \\
\text { subjetivo y la } \\
\text { espiritualidad en } \\
\text { pacientes con úlceras } \\
\text { venosas o úlceras de pie } \\
\text { diabético. }\end{array}$ & $\begin{array}{l}\text { Estudio } \\
\text { descriptivo, } \\
\text { prospectivo y } \\
\text { analítico. } \\
40 \text { pacientes } \\
\text { con úlceras } \\
\text { venosas y } 40 \\
\text { pacientes con } \\
\text { pie diabético }\end{array}$ & $\begin{array}{l}\text { - The Subjective } \\
\text { Wellbeing Scale } \\
\text { - Spirituality Self-Rating } \\
\text { Scale }\end{array}$ & $\begin{array}{l}\text { La mayoría de los pacientes consideran las } \\
\text { úlceras como un castigo y mostraron baja } \\
\text { espiritualidad. } \\
\text { Las puntuaciones medias en los } \\
\text { componentes de afecto positivo, afecto } \\
\text { negativo y satisfacción con la vida de } \\
\text { la Escala de Bienestar Subjetivo fueron } \\
2.55,1.55 \text { y } 1.50 \text {, respectivamente para } \\
\text { pacientes con úlceras venosas. }\end{array}$ \\
\hline
\end{tabular}


Tabla 1. Continuación...

\begin{tabular}{|c|c|c|c|c|}
\hline $\begin{array}{l}\text { Autores, } \\
\text { año, país } \\
\text { y nivel de } \\
\text { evidencia }\end{array}$ & Objetivo(s) & $\begin{array}{c}\text { Diseño } \\
\text { y } \\
\text { muestra }\end{array}$ & Instrumentos & $\begin{array}{l}\text { Hallazgos } \\
\text { principales }\end{array}$ \\
\hline $\begin{array}{l}\text { Chaby et } \mathrm{al}^{26} \text {. } \\
\text { (2013) } \\
\text { Francia } \\
\text { III }\end{array}$ & $\begin{array}{l}\text { Identificar las } \\
\text { características clínicas, } \\
\text { entre las que se } \\
\text { conocen como posibles } \\
\text { factores pronósticos } \\
\text { de curación de úlceras } \\
\text { venosas, y qué factores } \\
\text { sociodemográficos y } \\
\text { psicológicos asociados } \\
\text { a úlceras venosas, } \\
\text { se asocian con una } \\
\text { curación completa a la } \\
\text { semana } 24 \text {. }\end{array}$ & $\begin{array}{l}\text { Estudio } \\
\text { prospectivo, } \\
\text { multicéntrico, de } \\
\text { cohorte. } \\
104 \text { úlceras } \\
\text { venosas de } 104 \\
\text { pacientes }\end{array}$ & $\begin{array}{l}\text { - Beck Depression } \\
\text { Inventory } \\
\text { - Coopersmith Self- } \\
\text { Esteem Inventory }\end{array}$ & $\begin{array}{l}\text { Casi la mitad de los pacientes estaban } \\
\text { deprimidos y un tercio presentaron } \\
\text { baja autoestima. Sin embargo, no se } \\
\text { halló evidencia de asociación entre } \\
\text { estos factores psicológicos y la curación } \\
\text { completa de la úlcera venosa a la semana } \\
24 . \\
\text { En la semana } 24 \text { se curaron } 41 \text { de } 94 \\
\text { úlceras venosas. La curación completa } \\
\text { de las úlceras venosas se asoció } \\
\text { significativamente con cirugía superficial } \\
\text { de venas ( } P=0.001 \text { ), adherencia a la } \\
\text { terapia compresiva a la semana } 4 \text { ( } P=0.03 \text { ) } \\
\text { y a la semana } 24 \text { ( } P=0.01 \text { ), anquilosis } \\
\text { de la articulación del tobillo ( } P=0.01 \text { ) y } \\
\text { porcentaje medio de reducción del área de } \\
\text { la úlcera venosas a la semana } 4(P=0.04) \text {. }\end{array}$ \\
\hline $\begin{array}{l}\text { Salomé } \\
\text { et }^{335} \text {. } \\
\text { (2013) } \\
\text { Brasil } \\
\text { IV }\end{array}$ & $\begin{array}{l}\text { Evaluar los sentimientos } \\
\text { de impotencia en } \\
\text { pacientes con úlceras } \\
\text { venosas. }\end{array}$ & $\begin{array}{l}\text { Estudio } \\
\text { exploratorio, } \\
\text { descriptivo, } \\
\text { analítico } \\
\text { transversal. } \\
60 \text { pacientes }\end{array}$ & $\begin{array}{l}\text { Powerlessness } \\
\text { Assessment Tool }\end{array}$ & $\begin{array}{l}52 \% \text { de los pacientes informaron puntajes } \\
\text { de impotencia totales que varían de } 51 \text { a } \\
60 \text { y el } 32 \% \text { de ellos reportaron puntajes } \\
\text { totales de } 41 \text { a } 50 \text {, lo que indica que } \\
\text { estos pacientes tenían fuertes a muy } \\
\text { fuertes sentimientos de impotencia y falta } \\
\text { de control al enfrentar dificultades de la } \\
\text { vida diaria y aquellos relacionados con su } \\
\text { tratamiento. } \\
\text { La presencia de exudado y olor de la } \\
\text { herida tuvo un impacto significativo en los } \\
\text { sentimientos de impotencia ( } p=0.004 \text { ) en } \\
\text { la población del estudio. }\end{array}$ \\
\hline $\begin{array}{l}\text { Pereira } \\
\text { et al }{ }^{36} \text {. } \\
\text { (2014) } \\
\text { Brasil } \\
\text { IV }\end{array}$ & $\begin{array}{l}\text { Evaluar el bienestar } \\
\text { subjetivo y la depresión } \\
\text { en pacientes ancianos } \\
\text { con úlceras venosas. }\end{array}$ & $\begin{array}{l}\text { Estudio } \\
\text { descriptivo, } \\
\text { analítico y } \\
\text { multicéntrico. } \\
55 \text { pacientes } \\
\text { ancianos }\end{array}$ & $\begin{array}{l}\text { - Yesevage's reduced } \\
\text { version of the GDS-15 } \\
\text { - The Subjective Wellness } \\
\text { Scale }\end{array}$ & $\begin{array}{l}\text { 41.82\% de los pacientes tenían niveles } \\
\text { de depresión de leves a moderados, } \\
\text { 47.28\% mostraron depresión severa. En la } \\
\text { Escala subjetiva de bienestar, la mayoría } \\
\text { de los participantes se sienten infelices e } \\
\text { insatisfechos con la vida. }\end{array}$ \\
\hline $\begin{array}{l}\text { Edwards et } \mathrm{al}^{37} \text {. } \\
(2014) \\
\text { Australia } \\
\text { IV }\end{array}$ & $\begin{array}{l}\text { Identificar la prevalencia } \\
\text { y severidad de los } \\
\text { síntomas comunes y la } \\
\text { aparición de grupos de } \\
\text { síntomas en pacientes } \\
\text { con úlceras venosas. }\end{array}$ & $\begin{array}{l}\text { Análisis } \\
\text { secundario } \\
\text { de datos } \\
\text { obtenidos de } \\
\text { cuatro estudios } \\
\text { observacionales, } \\
\text { prospectivos } \\
\text { longitudinales. }\end{array}$ & $\begin{array}{l}\text { - Pain: The Medical } \\
\text { Outcomes Study Pain } \\
\text { Measures } \\
\text { - The Geriatric Depression } \\
\text { Scale of Yesavage. } \\
\text { - The Cardiff Wound } \\
\text { Impact Schedule } \\
\text { - Health-related quality } \\
\text { life: Short Form-12 Health } \\
\text { Survey Questionnaire } \\
\text { (SF-12) } \\
\text { - Fatigue: energy subscale } \\
\text { within the SF- } 12 \text {. }\end{array}$ & $\begin{array}{l}\text { Los síntomas más frecuentes fueron } \\
\text { trastornos del sueño (80\%), dolor (74\%) e } \\
\text { hinchazón de las extremidades inferiores } \\
\text { (67\%). } \\
\text { El 60\% de los pacientes informaron tres o } \\
\text { más síntomas a un nivel de intensidad de } \\
\text { moderado a severo. } \\
\text { Se identificaron dos grupos de síntomas: } \\
\text { (1) dolor, depresión, trastornos del sueño y } \\
\text { fatiga;(2) hinchazón, inflamación, exudado } \\
\text { y fatiga. }\end{array}$ \\
\hline
\end{tabular}


Tabla 1. Continuación...

\begin{tabular}{|c|c|c|c|c|}
\hline $\begin{array}{l}\text { Autores, } \\
\text { año, país } \\
\text { y nivel de } \\
\text { evidencia }\end{array}$ & Objetivo(s) & $\begin{array}{c}\text { Diseño } \\
y \\
\text { muestra }\end{array}$ & Instrumentos & $\begin{array}{l}\text { Hallazgos } \\
\text { principales }\end{array}$ \\
\hline $\begin{array}{l}\text { Salomé et } \\
a^{38} \text {. } \\
(2015) \\
\text { Brasil } \\
\text { IV }\end{array}$ & $\begin{array}{l}\text { Evaluar la asociación } \\
\text { de factores } \\
\text { sociodemográficos con } \\
\text { la esperanza para curar, } \\
\text { niveles de religiosidad } \\
\text { y la espiritualidad en } \\
\text { pacientes con úlceras } \\
\text { venosas. }\end{array}$ & $\begin{array}{l}\text { Estudio } \\
\text { descriptivo, } \\
\text { prospectivo, } \\
\text { analítico, clínico, } \\
\text { no aleatorizado. } \\
50 \text { pacientes. }\end{array}$ & $\begin{array}{l}\text { - Spirituality Self-rating } \\
\text { Scale orientation.scale. } \\
\text { Herth Hope Index }\end{array}$ & $\begin{array}{l}\text { Los pacientes presentaron niveles bajos } \\
\text { de espiritualidad (MD:10.20, DE: 4.35) } \\
\text { y moderada esperanza de curación } \\
\text { (MD:25.5, DE:8.40) respecto a sus úlceras } \\
\text { venosas. Valores más bajos de esperanza } \\
\text { frente a la curación se encontraron en } \\
\text { pacientes cuyas úlceras tenían exudado y } \\
\text { olor, tiempo entre } 20 \text { y } 39 \text { años y quienes } \\
\text { tenían la herida menos de un año. No } \\
\text { se identificaron asociaciones entre las } \\
\text { variables estudiadas. }\end{array}$ \\
\hline $\begin{array}{l}\text { Zhou y Jia }{ }^{39} \text {. } \\
\text { (2016) } \\
\text { Estados }\end{array}$ & $\begin{array}{l}\text { Investigar la prevalencia } \\
\text { de síntomas depresivos } \\
\text { en pacientes con } \\
\text { heridas y explorar la } \\
\text { relación entre depresión }\end{array}$ & $\begin{array}{l}\text { Estudio } \\
\text { descriptivo }\end{array}$ & $\begin{array}{l}\text { - The Patient Health } \\
\text { Questionnaire 9-item } \\
\text { (PHQ-9), (tool for } \\
\text { clinicians to the Primary }\end{array}$ & $\begin{array}{l}\text { La proporción de pacientes con síntomas } \\
\text { depresivos mínimos a severos fue } 80.8 \% \\
\text { en pacientes con úlceras venosas. El 26.6\% } \\
\text { de los pacientes tuvieron una detección } \\
\text { positiva para depresión. }\end{array}$ \\
\hline $\begin{array}{l}\text { Unidos } \\
\text { IV }\end{array}$ & $\begin{array}{l}\text { y características } \\
\text { sociodemográficas y } \\
\text { características de sus } \\
\text { heridas. }\end{array}$ & 222 pacientes. & $\begin{array}{l}\text { Care Evaluation of } \\
\text { Mental Disorders as } \\
\text { depression.) }\end{array}$ & $\begin{array}{l}\text { No se encontraron diferencias significativas } \\
\text { en las probabilidades de tener una } \\
\text { detección de depresión positiva y tener } \\
\text { una úlcera venosa y/o de cualquier } \\
\text { etiología. }\end{array}$ \\
\hline $\begin{array}{l}\text { Salomé et } \\
\mathrm{al}^{40} \text {. } \\
(2016) \\
\text { Brasil } \\
\text { IV }\end{array}$ & $\begin{array}{l}\text { Evaluar la autoestima y } \\
\text { la imagen corporal en } \\
\text { pacientes con úlceras } \\
\text { venosas. }\end{array}$ & $\begin{array}{l}\text { Estudio clínico } \\
\text { multicéntrico, } \\
\text { prospectivo, } \\
\text { descriptivo, } \\
\text { analítico. } \\
59 \text { pacientes }\end{array}$ & $\begin{array}{l}\text { - Body Investment Scale } \\
\text { - The Rosenberg Self- } \\
\text { esteem }\end{array}$ & $\begin{array}{l}\text { La puntuación media de autoestima } \\
\text { fue de 22,66 (DE:4.1) lo que indica baja } \\
\text { autoestima. El puntaje total promedio de la } \\
\text { Escala Body Investment Scale, fue de } 27.49 \\
\text { (DE:3.8), y los puntajes en las subescalas de } \\
\text { imagen corporal y tacto corporal también } \\
\text { fueron bajos, lo que indica sentimientos } \\
\text { negativos sobre el cuerpo. Existiendo una } \\
\text { asociación entre estas variables ( } P=0.002) \text {. }\end{array}$ \\
\hline $\begin{array}{l}\text { Parker et } a^{27} \text {. } \\
(2016) \\
\text { Australia } \\
\text { IV }\end{array}$ & $\begin{array}{l}\text { Identificar los factores } \\
\text { de riesgo asociados } \\
\text { con la curación tardía } \\
\text { en participantes con } \\
\text { úlceras venosas y, si los } \\
\text { factores psicosociales } \\
\text { juegan un papel en este } \\
\text { proceso. }\end{array}$ & $\begin{array}{l}\text { Estudio } \\
\text { de análisis } \\
\text { secundario de } \\
\text { siete estudios } \\
\text { previos } \\
\text { prospectivos } \\
\text { longitudinales. } \\
247 \text { pacientes } \\
\text { con } 318 \text { úlceras } \\
\text { venosas }\end{array}$ & $\begin{array}{l}\text { - The Short Form } 12 \text { - } \\
\text { item health survey } \\
\text { - The Geriatric } \\
\text { Depression Scale of } \\
\text { Yesavage. }\end{array}$ & $\begin{array}{l}\text { Cuatro predictores tempranos se } \\
\text { asociaron de manera independiente y } \\
\text { significativa con el fracaso de curación a las } \\
24 \text { semanas. Estos fueron: (1) participantes } \\
\text { que vivían solos (OR 2.3, IC 95\% [1.13- } \\
\text { 4.61], p = 0.03); (2) tener una reducción } \\
\text { de menos del 25\% en el área de la úlcera } \\
\text { dentro de las dos semanas de tratamiento } \\
\text { (OR 10.07, IC 95\% [4.60-22.19], p <0.001); } \\
\text { (3) tener puntuaciones más altas de } \\
\text { gravedad de la úlcera (OR 5.1, IC 95\% } \\
\text { [2.33-11.88], p = 0.001) y (4) participantes } \\
\text { que no fueron tratados con terapia de } \\
\text { compresión de alto nivel (es decir,> } 30 \\
\text { mmHg) en el momento de la valoración } \\
\text { inical (OR 4.18, IC 95\% [1.95-8.97], p = } \\
\text { 0.002). }\end{array}$ \\
\hline
\end{tabular}


Tabla 1. Continuación...

\begin{tabular}{|c|c|c|c|c|}
\hline $\begin{array}{l}\text { Autores, } \\
\text { año, país } \\
\text { y nivel de } \\
\text { evidencia }\end{array}$ & Objetivo(s) & $\begin{array}{c}\text { Diseño } \\
\text { y } \\
\text { muestra }\end{array}$ & Instrumentos & $\begin{array}{l}\text { Hallazgos } \\
\text { principales }\end{array}$ \\
\hline $\begin{array}{l}\text { Kouris et al }{ }^{41} \text {. } \\
(2016) \\
\text { Grecia } \\
\text { III }\end{array}$ & $\begin{array}{l}\text { Evaluar la calidad } \\
\text { de vida, ansiedad, } \\
\text { depresión, autoestima y } \\
\text { la soledad en pacientes } \\
\text { con úlceras en las } \\
\text { piernas. }\end{array}$ & $\begin{array}{l}\text { Estudio de casos } \\
\text { y controles } \\
102 \text { pacientes } \\
\text { con úlceras en } \\
\text { las piernas } \\
\text { Controles: } 102 \\
\text { voluntarios } \\
\text { sanos, } \\
\text { emparejados } \\
\text { por sexo y edad. }\end{array}$ & $\begin{array}{l}\text { - Dermatology Life } \\
\text { Quality IndexHospital } \\
\text {-Anxiety and Depression } \\
\text { Scale, - Rosenberg's Self- } \\
\text { esteem Scale } \\
\text { - The UCLA Loneliness } \\
\text { Scale }\end{array}$ & $\begin{array}{l}\text { Los pacientes con úlceras en las piernas } \\
\text { tuvieron puntuaciones estadísticamente } \\
\text { más altas en comparación con los } \\
\text { voluntarios sanos respecto al puntaje } \\
\text { total de la escala de ansiedad y depresión } \\
\text { y el puntaje de la subescala de ansiedad } \\
\text { ( } P=0.031 \text { y P = } 0.015 \text {, respectivamente). } \\
\text { Hubo diferencia significativa frente a las } \\
\text { puntuaciones de soledad, siendo mayor en } \\
\text { los casos que en los controles ( } P=0 \cdot 029 \text {. } \\
\text { Los pacientes femeninos presentaron } \\
\text { una puntuación más alta de ansiedad ( } P \\
=0 \cdot 027) \text { y aislamiento social ( } P=0 \cdot 048 \text { ), } \\
\text { y peor calidad de vida ( } P=0.018) \text { que los } \\
\text { pacientes masculinos con úlceras. }\end{array}$ \\
\hline $\begin{array}{l}\text { Walburn et } \\
\mathrm{al}^{28} \text {. } \\
\text { (2017) } \\
\text { Inglaterra } \\
\text { III }\end{array}$ & $\begin{array}{l}\text { Investigar el impacto del } \\
\text { estrés, las percepciones } \\
\text { de la enfermedad y los } \\
\text { comportamientos en la } \\
\text { curación de las úlceras } \\
\text { venosas. }\end{array}$ & $\begin{array}{l}\text { Estudio } \\
\text { prospectivo } \\
\text { observacional } \\
\text { por } 24 \text { semanas. } \\
63 \text { pacientes }\end{array}$ & $\begin{array}{l}\text { - The Perceived Stress } \\
\text { Scale } \\
\text { - Hospital Anxiety and } \\
\text { Depression Scale } \\
\text { - Revised IIIness } \\
\text { Perception } \\
\text { Questionnaire } \\
\text { - Health behavior of } \\
\text { Adapted Summary } \\
\text { of Diabetes Self-Care } \\
\text { Activities } \\
\text { - Adherence } \\
\text { Questionnaire } \\
\text { - Short-Form Health } \\
\text { Survey (SF-12) for quality } \\
\text { life. }\end{array}$ & $\begin{array}{l}\text { Una tasa de curación más lenta en el } \\
\text { área de la úlcera fue predicha por mayor } \\
\text { estrés (estandarizado } \beta=-0.61, p=.008 \text { ), } \\
\text { depresión (estandarizado } \beta=-0.51, p= \\
\text {.039) y mantener percepciones o creencias } \\
\text { negativas sobre la úlcera ( } \beta \text { estandarizado } \\
=-1.4, p=.045 \text { ). Una respuesta emocional } \\
\text { más negativa a la úlcera al inicio del } \\
\text { estudio (representación emocional de la } \\
\text { úlcera) se asoció con un mayor número de } \\
\text { semanas para sanar (hazard ratio [HR] = } \\
\text { 0,63, intervalo de confianza [IC] del 95\% = } \\
0,41-0,95, p=0.028 \text { ). }\end{array}$ \\
\hline $\begin{array}{l}\text { Finlayson } \\
\text { et al29. }^{29} \text { (2017) } \\
\text { Australia } \\
\text { IV }\end{array}$ & $\begin{array}{l}\text { Identificar subgrupos } \\
\text { de pacientes basado en } \\
\text { su experiencia con un } \\
\text { conjunto de síntomas } \\
\text { (dolor-depresión-fatiga- } \\
\text { trastorno del sueño) e } \\
\text { identificar las diferencias } \\
\text { en características de los } \\
\text { pacientes, así como la } \\
\text { curación de las úlceras } \\
\text { venosas y la calidad de } \\
\text { vida entre los grupos. }\end{array}$ & $\begin{array}{l}\text { Estudio } \\
\text { de análisis } \\
\text { secundario } \\
\text { de estudios } \\
\text { longitudinales. }\end{array}$ & $\begin{array}{l}\text { - Pain was measured } \\
\text { using the Medical } \\
\text { Outcomes } \\
\text { - Study Pain Measure } \\
\text { - The Geriatric } \\
\text { Depression Scale } \\
\text { - Fatigue was measured } \\
\text { with the SF-12 Vitality } \\
\text { Subscale } \\
\text { - Sleep disturbance } \\
\text { was measured with the } \\
\text { Cardiff Wound Impact } \\
\text { Schedule Disturbed Sleep } \\
\text { items. } \\
\text { - QOL index for Quality } \\
\text { Life }\end{array}$ & $\begin{array}{l}\text { 67\% de los pacientes estaban en un } \\
\text { subgrupo de síntomas leves (es decir, } \\
\text { que no experimentaban o de forma } \\
\text { leve experimentaron dolor, síntomas } \\
\text { depresivos, fatiga o trastornos del sueño). } \\
33 \% \text { de los pacientes se clasificaron } \\
\text { en el subgrupo de síntomas severos y } \\
\text { presentaron puntajes de calidad de vida } \\
\text { más pobres ( } \mathrm{t}=8.06, \mathrm{P}<0.001 \text { ) y tenían } \\
1.5 \text { veces menos (IC } 95 \% \text { 1.02-2.08) } \\
\text { probabilidades de sanar en las siguientes } \\
24 \text { semanas ( } P=0.037 \text { ). }\end{array}$ \\
\hline
\end{tabular}




\section{DISCUSIÓN}

Siete fueron los factores psicosociales identificados en este grupo poblacional a partir de la literatura revisada. Depresión, ansiedad, sentimientos de impotencia, bienestar subjetivo, autoestima, soledad y espiritualidad-esperanza de cura.

La depresión fue el factor más común presente en este grupo de población ${ }^{30,31,33,36,37,39,41}$ Moffat et a ${ }^{130}$, en su estudio de casos y controles identificó que los pacientes con UV tenían niveles de depresión significativamente mayores, menos redes de apoyo y un menor soporte social percibido que los controles, además, la calidad de vida de los primeros era significativamente menor en todos los dominios considerados, dolor corporal, sueño, aislamiento social, respuesta emocional, energía y movilidad. El origen de ciertos estados emocionales negativos podría explicarse en el hecho de lo que implica vivir con una UV, generan dolor, limitación en la movilidad, exudado y muchas veces olor desagradable lo cual ineludiblemente repercute en el bienestar de las personas.

De hecho, el olor, el exudado y el dolor son aspectos asociados en varios estudios no sólo con los estados de depresión y ansiedad, sino también, con sentimientos de impotencia, bajo bienestar subjetivo y baja espiritualidad ${ }^{34,38,40,42}$. A1 respecto, Aguiar et al ${ }^{43}$, indica que los pacientes con UV son víctimas de los preconceptos, la discriminación y la vergüenza social por presentar en su cuerpo algo que los vuelve diferentes a las demás personas. Las personas con UV se perciben restringidas porque creen que pueden incomodar a otras personas, sentimiento de segregación que dificulta la interacción social y repercute en el aislamiento social y la baja autoestima. Hopkins ${ }^{44}$ a su vez, describe que los pacientes con UV evidencian una disrupción biográfica, es decir, hay una fragmentación en el curso de la vida antes y después de una UV.

Por otro lado, se informa que los síntomas depresivos son comunes en pacientes con UV $\geq 90$ días y con dolor asociado, Zhou y Jia ${ }^{39}$ informaron que las probabilidades de una prueba positiva para depresión en pacientes con heridas $>90$ días a partir del examen inicial, fue de 3.20 (IC95\% $[1.49,6.87])$ y en aquellos con dolor relacionado con la úlcera era de 2.53 (IC95\% [1.26, 5.08]). De hecho, la depresión hizo parte del grupo de síntomas experimentados más frecuentes en pacientes australianos con UV, junto con el dolor, trastornos del sueño y la fatiga. El aumento de la severidad de estos sistemas se asoció con el género femenino, el antecedente de artritis reumatoide, la mayor duración de la úlcera y una disminución de la calidad de vida ${ }^{37}$. Respecto a la ansiedad, esta se ha asociado con la presencia de olor, dolor y exudado ${ }^{42,45}$; desde la evidencia cualitativa los pacientes asocian la ansiedad con el miedo a caerse o a sufrir una lesión que podría conducir al desarrollo de UV adicionales, como también a que la herida empeore o se infecte ${ }^{46}$. A su vez, los sentimientos de impotencia cobran relevancia debido a la falta de control percibido sobre la UV, los pacientes han descrito esto como un "proceso de curación eterna" ${ }^{47}$. En los adultos mayores, la movilidad limitada, la disminución de la capacidad funcional y el aislamiento auto impuesto de la situación son condiciones que pueden exacerbar este tipo de sentimientos que deterioran aún más el bienestar y la calidad de vida ${ }^{48}$.

Bienestar subjetivo ${ }^{34,36}$, autoestima ${ }^{32,40}$, soledad $^{41} \mathrm{y}$ espiritualidad ${ }^{34,38}$ fueron otros factores hallados, pero en menor medida. Hallazgos que dejan de manifiesto probablemente dos aspectos, el primero, que en la literatura se ha dado mayor relevancia al estudio de las emociones o estados negativos que a las emociones positivas (bienestar, esperanza, optimismo, autoestima, resiliencia, etc.). Y segundo, estudios en otras condiciones crónicas han demostrado el efecto protector que las emociones positivas tienen en los comportamientos de salud y en los resultados clínicos de los pacientes. Por ejemplo, en la diabetes tipo 2, los estados psicológicos positivos, como el bienestar, se asocian prospectivamente con una HbA1c más baja, menos complicaciones de la diabetes y disminución de eventos cardiacos y mortalidad ${ }^{49}$.

\section{Asociación de los factores psicosociales con la curación de las UV}

Niveles altos de estrés, depresión y una percepción negativa hacia la herida se asociaron con una tasa de cambio significativamente más lenta en el área de la UV independientemente de las características sociodemográficas, comorbilidades y características de la úlcera ${ }^{28}$. Parker et a $\mathrm{a}^{27}$. explicó que los participantes que vivían solos tenían el doble de probabilidades de retraso en la curación que aquellos que no vivían solos. Finlayson et a $\mathrm{l}^{29}$, por su parte, evidenciaron que los pacientes clasificados en el grupo de experiencia de síntomas severos (dolor, depresión, trastorno del sueño y fatiga) tuvieron un tiempo significativamente más largo de 
curación. Estos pacientes tardaron una mediana de 24 semanas para curar después del inicio del estudio, en comparación a 16 semanas para quienes habían sido clasificados en el grupo de síntomas leves. Adicionalmente, determinó que estos pacientes tenían significativamente menos probabilidades de curarse después de controlar el tratamiento basado en la evidencia, es decir, la terapia de compresión y la gravedad de la úlcera, este mismo grupo al inició del estudió tenían 1.5 veces menos probabilidades de sanar en las siguientes 24 semanas (IC:95\%, 1.02-2.08; $\mathrm{p}=0.037)^{29}$.

Existe evidencia que demuestra como el estrés y las emociones negativas, depresión y ansiedad, además de tener un efecto negativo sobre la tasa de cicatrización de las heridas, regula negativamente la respuesta proinflamatoria sistémica $^{50}$. Se ha comprobado la correlación negativa entre estrés percibido, distrés emocional, curación de heridas y producción de cortisol cuyos niveles aumentan a medida que las heridas demoran más en su proceso de cicatrización ${ }^{51}$. Glaser y colaboradores, establecieron que puntuaciones altas de estrés percibido evidenciaron significativamente bajos niveles de interleucina 1 y 8 necesarias para regular la producción, liberación y activación de metaloproteinasas importantes en la reconstrucción y remodelación de las heridas ${ }^{52}$. Como también se ha comprobado el efecto positivo de las intervenciones que buscan disminuir el estrés psicológico en la curación de las heridas. Metaanálisis y revisiones sistemáticas de literatura señalan que intervenciones como el ejercicio físico, la escritura expresiva, la hipnosis, soporte social, relajación, yoga, han producido un efecto positivo sobre aspectos como la depresión, la ansiedad, tasas de cicatrización y factores de la respuesta inmune ${ }^{53,54}$.

De hecho, Enfermería a través de estudios experimentales en el área ha evidenciado que los pacientes que reciben atención bajo un modelo de cuidado comunitario que promueve la interacción social, la participación, la empatía, el apoyo entre compañeros y el empoderamiento de su condición han evidenciado tiempos de cicatrización más cortos, mejora en la calidad de vida, soporte social percibido, autoestima y disminución del dolor ${ }^{55,56}$. Más aún, las intervenciones de enfermería que fomentan los estilos de vida saludable, el autocuidado, la actividad física y el ejercicio también han mostrado resultados positivos en las tasas de cicatrización de las UV entre otras variables psicosociales ${ }^{57-59}$.

Dentro de las limitaciones de esta revisión se encuentra la generalización de los hallazgos, debido al limitado número de estudios identificados específicamente con pacientes con
UV, en su mayoría desarrollados en Brasil desconociéndose de alguna manera el comportamiento que estos factores podrían tener en otras culturas. El nivel de evidencia aún es descriptivo, la mayoría de las muestras fueron por conveniencia y pequeñas.

\section{Implicaciones para la práctica de enfermería}

La revisión sugiere una urgente necesidad de mejorar la evidencia disponible frente a los factores psicosociales presentes en los pacientes con UV y su asociación en los procesos de curación. La valoración de aspectos emocionales negativos y positivos deben ser introducidos como un componente central y principal en la valoración de enfermería. La propuesta y desarrollo de intervenciones de enfermería con participación multidisciplinar en unidades de consulta externa y centros comunitarios debe ser una prioridad. Tales intervenciones además de promover estilos de vida saludables, deben propender por el bienestar de las personas, potencializar otros factores psicosociales como la resiliencia, la esperanza, la espiritualidad, la gratitud, entre otros, ya que estos podrían contribuir a la adopción, modificación y/o ejecución de ciertos comportamientos saludables que, a su vez, coadyuvarían en el manejo de los síntomas de la UV e incluso en su proceso de curación.

\section{CONCLUSIONES}

Esta revisión integradora pone de manifiesto que la depresión es uno de los factores psicológicos medido con mayor frecuencia en esta población. La evidencia disponible frente a la medición de diferentes aspectos psicológicos y sociales presentes en los pacientes con UV y su asociación con la curación es escasa. Se requiere el desarrollo juicioso de estudios comparativos, de cohorte, retrospectivos, prospectivos, longitudinales y de intervención que permitan comprender de una mejor forma este fenómeno de interés para enfermería con el objeto de proporcionar un cuidado que responda a las necesidades reales de los pacientes. La exploración de estados psicológicos positivos como optimismo, resiliencia, esperanza, entre otros, desde los paradigmas de investigación cualitativo y cuantitativo constituye un reto actual para Enfermería. 
AGRADECIMIENTOS

La primera autora agradece a la Dirección Académica de la Universidad Nacional de Colombia, Sede Bogotá por

la beca otorgada para sus estudios de Doctorado.
CONTRIBUCIÓN DE LOS AUTORES

\author{
Conceitualização, Cifuentes,JE; Metodologia, Cifuentes,
} JE; Investigação, Cifuentes, JE, Guerrero, S; Redação Primeira versão, Cifuentes,JE; Redação - Revisão \& Edição, Cifuentes, JE; Guerrero, S; Supervisão, Guerrero, S.

\section{REFERENCIAS}

1. Asociación Española de Enfermería Vascular y Heridas. Guía de Práctica Clínica. Consenso sobre úlceras vasculares y pie diabético de la Asociación Española de Enfermería Vascular y Heridas (AEEVH). Madrid: Asociación Española de Enfermería Vascular y Heridas; 2017.

2. Onida S, Davies AH. Predicted burden of venous disease. Phlebology. 2016;31(1 Suppl):74-9. https://doi. org/10.1177/0268355516628359

3. Marinel.lo Roura J, Verdú Soriano J. Conferencia Nacional De Consenso Sobre Las Úlceras De La Extremidad Inferior (C.O.N.U.E.I.) [Internet]. Segunda. Ergon, editor. Madrid, España; 2018. 146 p. [Citado en: 03 Dec. 2019. Disponible en: https://gneaupp.info/conferencia-nacional-de-consensosobre-las-ulceras-de-la-extremidad-inferior-c-o-n-u-e-i/

4. Nettel F, Rodríguez N, Nigro J, González M, Conde A, Muñoa A, et al. Primer consenso latinoamericano de úlceras venosas: Resumen. Rev Mex Angiol. 2013;41(3):95-126.[Citado en: 12 Nov. 2019]. Disponible en: http://www.rmangiologia.com/

5. Kelechi TJ, Johnson JJ, Yates S. Chronic venous disease and venous leg ulcers: an evidence-based update. J Vasc Nurs. 2015;33(2):36-46. https://doi.org/10.1016/j.jvn.2015.01.003

6. Franks PJ, Barker J, Collier M, Gethin G, Haesler E, Jawien A, et al. Management of patients with venous leg ulcers: challenges and current best practice. J Wound Care. 2016;25(6 Suppl):24-6. https://doi.org/10.12968/ jowc.2016.25.Sup6.S1

7. Grey M, Schulman-Green D, Knafl K, Reynolds NR. A revised self- and family management framework. Nurs Outlook. 2015;63(2):162-70. https://doi.org/10.1016/j. outlook.2014.10.003

8. Alavi A, Sibbald RG, Phillips TJ, Miller OF, Margolis DJ, Marston W, et al. What's new: management of venous leg ulcers. J Am Acad Dermatol. 2016;74(4):627-40. https://doi. org/10.1016/j.jaad.2014.10.048

9. Finlayson $\mathrm{K}, \mathrm{Wu} \mathrm{M}-\mathrm{L}$, Edwards HE. Identifying risk factors and protective factors for venous leg ulcer recurrence using a theoretical approach: a longitudinal study. Int J Nurs Stud. 2015;52(6):1042-51. https://doi.org/10.1016/j. ijnurstu.2015.02.016

10. Finlayson K, Edwards HE, Courtney M. Factors associated with recurrence of venous leg ulcers: a survey and retrospective chart review. Int J Nurs Stud. 2009;46(8):10718. https://doi.org/10.1016/j.ijnurstu.2008.12.012

11. Finlayson K, Edwards HE, Courtney M. Relationships between preventive activities, psychosocial factors and recurrence of venous leg ulcers: a prospective study. J Adv Nurs. 2011;67(10):2180-90. https://doi.org/10.1111/j.13652648.2011.05653.x

12. González-Consuegra RV, Verdú J. Quality of life in people with venous leg ulcers: an integrative review. J Adv Nurs. 2011;67(5):926-44. https://doi.org/10.1111/j.13652648.2011.05653.x

13. Phillips P, Lumley E, Duncan R, Aber A, Woods HB, Jones $G L$, et al. A systematic review of qualitative research into people's experiences of living with venous leg ulcers. J Adv Nurs. 2017;74(3):550-63. https://doi.org/10.1111/jan.13465

14. Stechmiller JK, Lyon D, Schultz G, Gibson DJ, Weaver MT, Wilkie D, et al. Biobehavioral mechanisms associated with nonhealing wounds and psychoneurologic symptoms (pain, cognitive dysfunction, fatigue, depression, and anxiety) in older individuals with chronic venous leg ulcers. Biol Res Nurs. 2019;21(4):407-19. https://doi. org/10.1177/1099800419853881

15. Herber OR, Schnepp W, Rieger MA. A systematic review on the impact of leg ulceration on patients' quality of life. Health Qual Life Outcomes. 2007;5:44. https://doi. org/10.1186/1477-7525-5-44

16. Gouin J-P, Kiecolt-Glaser JK. The impact of psychological stress on wound healing: methods and mechanisms. Immunol Allergy Clin. 2011;31(1):81-93. https://doi. org/10.1016/j.iac.2010.09.010

17. Bosch JA, Engeland CG, Cacioppo JT, Marucha PT. Depressive symptoms predict mucosal wound healing. Psychosom Med. 2007;69(7):597-605. https://doi.org/10.1097/ PSY.0b013e318148c682

18. Doering LV, Moser DK, Lemankiewicz W, Luper C, Khan S. Depression, healing, and recovery from coronary artery bypass surgery. Am J Crit Care. 2005;14(4):316-23. https:// doi.org/10.4037/ajcc2005.14.4.316

19. Cole-King A, Harding KG. Psychological factors and delayed healing in chronic wounds. Psychosom Med. 2001;63(2):21620. https://doi.org/10.1097/00006842-200103000-00004 
20. Moffatt CJ, Franks PJ, Doherty DC, Smithdale R, Martin R. Sociodemographic factors in chronic leg ulceration. $\mathrm{Br}$ J Dermatol. 2006;155(2):307-12. https://doi.org/10.1111/ j.1365-2133.2006.07265.x

21. Wounds International. International Best Practice Statement: optimising patient involvement in wound management [Internet]. London; 2016. [Citado en: 18 Nov. 2019]. Disponible en: https://www.woundsinternational.com

22. Wounds UK. Best Practice Statement: Holistic Management of venous leg ulceration [Internet]; 2016 [Citado en: 18 Nov. 2019]. Disponible en: https://www.wounds-uk.com

23. Soares CB, Hoga LAK, Peduzzi M, Sangaleti C, Yonekura T, Silva DRAD. Revisión integradora: conceptos y métodos utilizados en enfermería. Rev Esc Enferm USP. 2014;48(2):329-39. https://doi.org/10.1590/S0080-6234201400002000020

24. LoBiondo-Wood G, Haber J. Nursing research: methods and critical appraisal for evidence-based practice. Amsterdam: Mosby Elsevier; 2018.

25. Joanna Briggs Institute [Internet]. The Joanna Briggs Institute. 2015. [Citado en: 08 Sep. 2019]. Disponible en: https://joannabriggs.org/

26. Chaby G, Senet P, Ganry O, Caudron A, Thuillier D, Debure C, et al. Prognostic factors associated with healing of venous leg ulcers: a multicentre, prospective, cohort study. $\mathrm{Br}$ J Dermatol. 2013;169(5):1106-13. https://doi.org/10.1111/ bjd. 12570

27. Parker CN, Finlayson KJ, Edwards HE. Ulcer area reduction at 2 weeks predicts failure to heal by 24 weeks in the venous leg ulcers of patients living alone. J Wound Care. 2016;25(11):626-34. https://doi.org/10.12968/ jowc.2016.25.11.626

28. Walburn J, Weinman J, Norton S, Hankins M, Dawe K, Banjoko $\mathrm{B}$, et al. Stress, illness perceptions, behaviors, and healing in venous leg ulcers findings from a prospective observational study. Psychosom Med. 2017;79(5):585-92. https://doi. org/10.1097/PSY.0000000000000436

29. Finlayson K, Miaskowski C, Alexander K, Liu W-H, Aouizerat B, Parker C, et al. Distinct wound healing and quality-of-life outcomes in subgroups of patients with venous leg ulcers with different symptom cluster experiences. J Pain Symptom Manag. 2017;53(5):871-9. https://doi.org/10.1016/j. jpainsymman.2016.12.336

30. Moffatt CJ, Franks PJ, Doherty DC, Smithdale R, Steptoe A. Psychological factors in leg ulceration: a case-control study. Br J Dermatol. 2009;161(4):750-6. https://doi.org/10.1111/ j.1365-2133.2009.09211.x

31. Nogueira GS, Zanin CR, Miyazaki MCOS, Godoy JMP. Venous leg ulcers and emotional consequences. Int J Low Extrem Wounds. 2009;8(4):194-6. https://doi. org/10.1177/1534734609350548

32. Faria E, Blanes L, Hochman B, Mesquita Filho M, Ferreira L. Health-related quality of life, self- esteem, and functional status of patients with leg ulcers. Wounds. 2011;23(1):4-10.
33. Salomé GM, Blanes L, Ferreira LM. Avaliação de sintomas depressivos em pessoas com úlcera venosa. Rev Bras Cir Plást. 2012;27(1):124-9. https://doi.org/10.1590/S198351752012000100021

34. Salomé GM, Pereira VR, Ferreira LM. Spirituality and subjective wellbeing in patients with lower-limb ulceration. J Wound Care. 2013;22(5):230-6. https://doi.org/10.12968/ jowc.2013.22.5.230

35. Salomé GM, Openheimer DG, Almeida SA, Bueno MLGB, Dutra RAA, Ferreira LM. Feelings of powerlessness in patients with venous leg ulcers. J Wound Care. 2013;22(11):628-34. https://doi.org/10.12968/jowc.2013.22.11.628

36. Pereira RC, Santos EF, Queiroz MA, Massahud Junior MR, Carvalho MRF, Salomé GM. Depression and wellness in elderly patients with venous ulcers. Rev Bras Cir Plást. 2014;29(4):567-74. https://doi.org/10.5935/21771235.2014RBCP0099

37. Edwards $H E$, Finlayson $K$, Skerman $H$, Alexander $K$, Miaskowski C, Aouizerat B, et al. Identification of symptom clusters in patients with chronic venous leg ulcers. J Pain Symptom Manag. 2014;47(5):867-75. https://doi. org/10.1016/j.jpainsymman.2013.06.003

38. Salomé GM, Almeida SA, Ferreira LM. Association of sociodemographic factors with hope for cure, religiosity, and spirituality in patients with venous ulcers. Adv Skin Wound Care. 2015;28(2):76-82. https://doi.org/10.1097/01. ASW.0000459844.07689.02

39. Zhou K, Jia P. Depressive symptoms in patients with wounds: a cross-sectional study. Wound Repair Regen. 2016;24(6):1059-65. https://doi.org/10.1111/wrr.12484

40. Salomé GM, Almeida SA, Pereira MTJ, Massahud Junior MR, Moreira CNO, Brito MJA, et al. The impact of venous leg ulcers on body image and self-esteem. Adv Skin Wound Care. 2016;29(7):316-21. https://doi.org/10.1097/01. ASW.0000484243.32091.0c

41. Kouris A, Armyra K, Christodoulou C, Sgontzou T, Karypidis D, Kontochristopoulos G, et al. Quality of life psychosocial characteristics in Greek patients with leg ulcers: a case control study. Int Wound J. 2016;13(5):744-7. https://doi. org/10.1111/iwj.12363

42. Jones JE, Robinson J, Barr W, Carlisle C. Impact of exudate and odour from chronic venous leg ulceration. Nurs Stand. 2008;22(45):53-61. https://doi.org/10.7748/ ns2008.07.22.45.53.c6592

43. Aguiar ACSA, Sadigursky D, Martins LA, Menezes TMO, Santos ALS, Reis LA. Repercusión social experimentado por anciano con úlcera venosa. Rev Gaúcha Enferm. 2016;37(3):1-6. https://doi.org/10.1590/1983-1447.2016.03.55302

44. Hopkins A. Disrupted lives: investigating coping strategies for non-healing leg ulcers. Br J Nurs. 2004;13(9):556-63. https://doi.org/10.12968/bjon.2004.13.9.12972

45. Jones J, Barr W, Robinson J, Carlisle C. Depression in patients with chronic venous ulceration. Br J Nurs. 2006;15(11):S1723. 
46. Stewart A, Edwards HE, Finlayson K. Reflection on the cause and avoidance of recurrent venous leg ulcers: an interpretive descriptive approach. J Clin Nurs. 2018;27(56):e931-9. https://doi.org/10.1111/jocn.13994

47. Chase SK, Melloni M, Savage A. A forever healing: the lived experience of venous ulcer disease. J Vasc Nurs. 1997;15(2):73-8. https://doi.org/10.1016/S10620303(97)90004-2

48. Ebbeskog B, Ekman S-L. Elderly persons' experiences of living with venous leg ulcer: living in a dialectal relationship between freedom and imprisonment. Scand J Caring Sci. 2001;15(3):235-43. https://doi.org/10.1046/j.1471$6712.2001 .00018 . x$

49. Huffman JC, DuBois CM, Millstein RA, Celano CM, Wexler D. Positive psychological interventions for patients with type 2 diabetes: rationale, theoretical model, and intervention development. J Diabetes Res. 2015;2015:428349. https:// doi.org/10.1155/2015/428349

50. Walburn J, Vedhara K, Hankins $M$, Rixon L, Weinman J. Psychological stress and wound healing in humans: a systematic review and meta-analysis. I Psychosom Res. 2009;67(3):253-71. https://doi.org/10.1016/j. jpsychores.2009.04.002

51. Ebrecht M, Hextall J, Kirtley L-G, Taylor A, Dyson M, Weinman J. Perceived stress and cortisol levels predict speed of wound healing in healthy male adults. Psychoneuroendocrinology. 2004;29(6):798-809. https://doi.org/10.1016/S03064530(03)00144-6

52. Glaser R, Kiecolt-Glaser JK, Marucha PT, MacCallum RC, Laskowski BF, Malarkey WB. Stress-related changes in proinflammatory cytokine production in wounds. Arch Gen Psychiatry. 1999;56(5):450-6. https://doi.org/10.1001/ archpsyc.56.5.450
53. Moraes LJ, Miranda MB, Loures LF, Mainieri AG, Mármora CHC. A systematic review of psychoneuroimmunologybased interventions. Psychol Heal Med. 2017;23(6):635-52. http://doi.org/10.1080/13548506.2017.1417607

54. Robinson $H$, Norton S, Jarrett $P$, Broadbent E. The effects of psychological interventions on wound healing: a systematic review of randomized trials. $\mathrm{Br} J$ Health Psychol. 2017;22(4):805-35. https://doi.org/10.1111/bjhp.12257

55. Edwards HE, Courtney M, Finlayson K, Lewis C, Lindsay E, Dumble J. Improved healing rates for chronic venous leg ulcers: Pilot study results from a randomized controlled trial of a community nursing intervention. Int J Nurs Pract. 2005;11(4):169-76. https://doi.org/10.1111/j.1440172X.2005.00521.x

56. Edwards HE, Courtney M, Finlayson K, Shuter P, Lindsay E. A randomised controlled trial of a community nursing intervention: improved quality of life and healing for clients with chronic leg ulcers. J Clin Nurs. 2009;18(11):1541-9. https://doi.org/10.1111/j.1365-2702.2008.02648.x

57. Domingues EAR, Kaizer UAO, Lima MHM. Effectiveness of the strategies of an orientation programme for the lifestyle and wound-healing process in patients with venous ulcer: a randomised controlled trial. Int Wound J. 2018;15(5):798806. https://doi.org/10.1111/iwj.12930

58. O'Brien J, Finlayson K, Kerr G, Edwards HE. Evaluating the effectiveness of a self-management exercise intervention on wound healing, functional ability and health-related quality of life outcomes in adults with venous leg ulcers: a randomised controlled trial. Int Wound J. 2016;14(1):130-7. https://doi.org/10.1111/iwj.12571

59. Tavares APC, Chaves Sá SP. Cuidados de enfermería en la promoción del autocuidado de los pacientes con úlceras en las piernas: una revisión integradora. Cul Cuid. 2017;21(48):168-77. https://doi.org/10.14198/ cuid.2017.48.19 